\title{
Pathologically Proven Spontaneous Remission of IgG4-related Retroperitoneal Fibrosis
}

\author{
Hideaki Yamakawa ${ }^{1,2}$, Akimasa Sekine ${ }^{1}$, Yumie Yamanaka ${ }^{1,2}$, Shinko Sadoyama ${ }^{1}$, \\ Tomohisa Baba ${ }^{1}$, Eri Hagiwara ${ }^{1}$, Koji Okudela ${ }^{3}$ and Takashi Ogura ${ }^{1}$
}

\begin{abstract}
Some forms of idiopathic retroperitoneal fibrosis (RF) have recently been considered to be a part of the spectrum of immunoglobulin G4 (IgG4)-related disease. This case report is the first description of a spontaneous remission in a patient with pathologically proven IgG4-related RF. Although the pathogenesis and longterm disease behavior of IgG4-related RF remains unknown, we believe that an initial assessment consisting of only careful monitoring might be one important strategy, especially in asymptomatic IgG4-related RF patients without nephropathy, while carefully monitoring these patients for the risk of recurrence.
\end{abstract}

Key words: retroperitoneal fibrosis, spontaneous remission, IgG4-related disease

(Intern Med 56: 1867-1871, 2017)

(DOI: 10.2169/internalmedicine.56.7996)

\section{Introduction}

Retroperitoneal fibrosis (RF) is a rare disease characterized by the development of inflammation and fibrosis in the soft tissues of the retroperitoneum and other abdominal organs (1). The radiologic findings of a soft-tissue mass surrounding the abdominal aorta and the iliac arteries, with the possible encasement of neighboring structures, such as the ureters and the inferior vena cava, usually suggests a diagnosis of RF (2). Recent reports have shown that to some extent, that cases of idiopathic RF included immunoglobulin G4 (IgG4)-related RF (3). However, the concept of IgG4related disease is relatively new, and few studies have so far been published on this disease. Patients with RF and associated with clinical symptoms (e.g., fatigue, weight loss, abdominal or back pain) or those with hydronephrosis and acute renal failure caused by ureteral obstruction usually require steroid therapy, regardless of the cause of RF (4). Therefore, the natural course of this disease remains unknown. We herein report a pathologically proven case of a spontaneous remission of IgG4-related RF.

\section{Case Report}

A 72-year-old man who was an ex-smoker (3 packs/day for 42 years) had no known history of dust exposure including asbestos. He had a medical history of cerebral infarction 10 years perviously and chronic kidney disease (CKD) [estimated glomerular filtration rate (eGFR) of $46.8-57.1 \mathrm{~mL} /$ $\mathrm{min} / 1.73 \mathrm{~m}^{2}$ for the past 2 years]. He initially presented with complaints of fever, cough, and right-sided chest pain for one week and was then referred to our hospital.

The physical examination on admission did not reveal any eruptions or swelling of joints, superficial lymph nodes, lacrimal glands, or salivary glands. His body temperature was elevated at $38.6^{\circ} \mathrm{C}$. An enhanced computed tomography (CT) scan revealed slight bilateral pleural effusion, a soft tissue mass surrounding the abdominal aorta, and bilateral hydronephrosis caused by sequential thickening of the retroperitoneum with encasement of both ureters, which were suspected to indicate RF (Fig. 1A-C). No other abnormalities, such as lymphadenopathy or abnormal kidney findings (e.g., multiple low-density areas, hypovascular solitary mass, hypertrophic renal pelvic wall) were revealed. The laboratory findings were as follows: leukocyte count $11,000 /$

${ }^{1}$ Department of Respiratory Medicine, Kanagawa Cardiovascular and Respiratory Center, Japan, ${ }^{2}$ Department of Respiratory Medicine, Tokyo Jikei University Hospital, Japan and ${ }^{3}$ Department of Pathobiology, Yokohama City University Graduate School of Medicine, Japan Received for publication July 14, 2016; Accepted for publication January 10, 2017 Correspondence to Dr. Hideaki Yamakawa, hide1144@jikei.ac.jp 

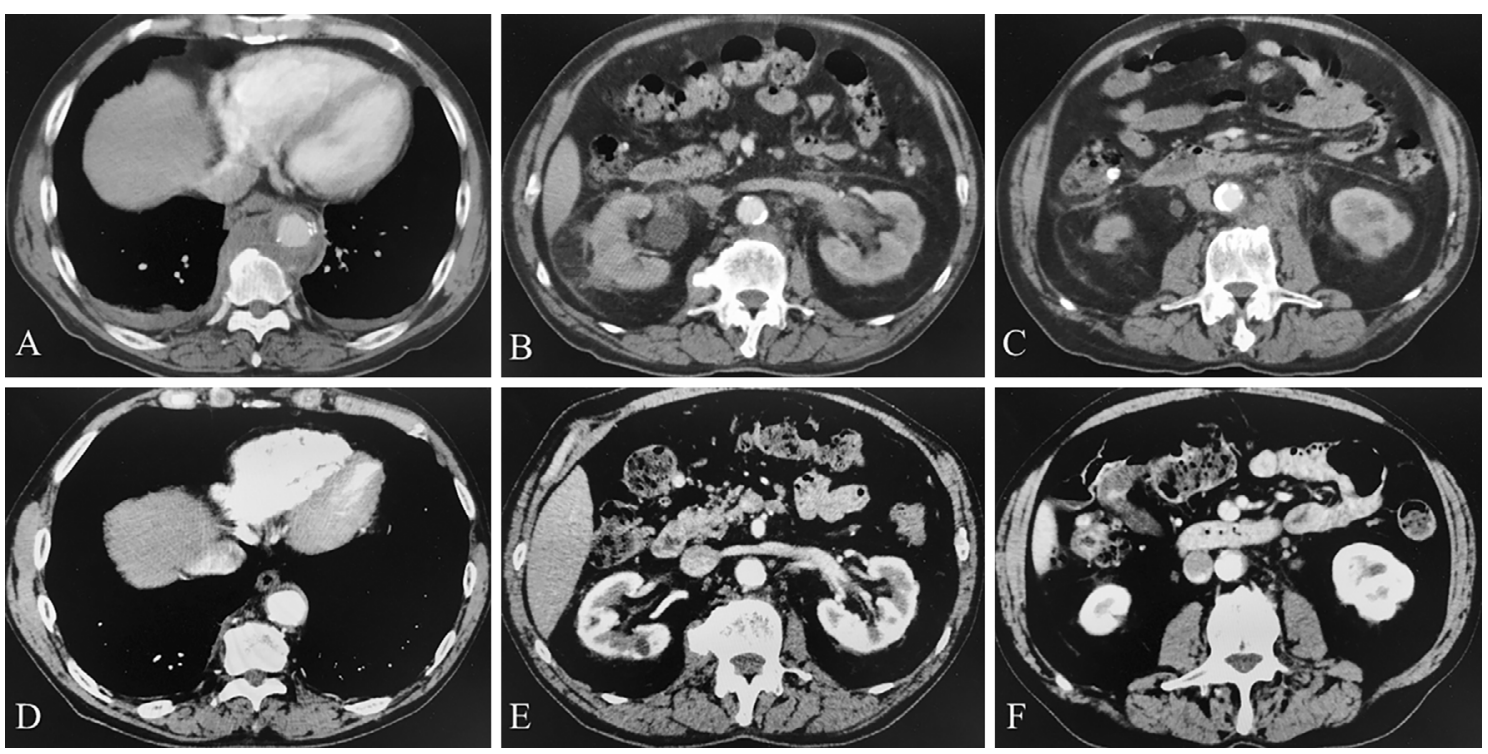

Figure 1. Computed tomography (CT) performed at initial presentation (A-C) and 2 months after the diagnosis of IgG4-related retroperitoneal fibrosis (RF) (D-F). (A-C): CT on admission showed bilateral pleural effusion, a soft tissue mass surrounding the abdominal aorta, and bilateral hydronephrosis caused by sequential thickening of the retroperitoneum with encasement of both ureters. (DF): Two months after the diagnosis of IgG4-related RF, CT showed improvement of the retroperitoneal lesions.

$\mu \mathrm{L}(74.7 \%$ neutrophils, $12.5 \%$ lymphocytes, $9.5 \%$ monocytes, and $3.1 \%$ eosinophils), hemoglobin $13.1 \mathrm{~g} / \mathrm{dL}$, thrombocytes $37.5 \times 10^{4} / \mu \mathrm{L}$, creatinine $1.01 \mathrm{mg} / \mathrm{dL}$ (eGFR of 56.2 $\mathrm{mL} / \mathrm{min} / 1.73 \mathrm{~m}^{2}$ ), total bilirubin $0.4 \mathrm{mg} / \mathrm{dL}$, and elevated levels of aspartate aminotransferase of $62 \mathrm{IU} / \mathrm{L}$, alanine aminotransferase of $61 \mathrm{IU} / \mathrm{L}$, gamma-glutamyl transpeptidase of $91 \mathrm{IU} / \mathrm{L}$, alkaline phosphatase of $404 \mathrm{IU} / \mathrm{L}$, lactate dehydrogenase of $255 \mathrm{IU} / \mathrm{L}, \mathrm{C}$-reactive protein (CRP) of $18.16 \mathrm{mg} / \mathrm{dL}$, erythrocyte sedimentation rate of $114 \mathrm{~mm} / \mathrm{h}$, immunoglobulin $\mathrm{G}(\mathrm{IgG})$ of $2,515 \mathrm{mg} / \mathrm{dL}, \mathrm{IgG} 4 \mathrm{of} 185 \mathrm{mg} /$ $\mathrm{dL}$, and soluble interleukin (IL)-2 receptor of 1,490 U/mL. IL-6 was not elevated at $<8 \mathrm{pg} / \mathrm{mL}$. Anti-nuclear antibody was less than 40 titers according to immunofluorescence testing, and no other autoantibodies including anti-SS-A, anti-aminoacyl tRNA synthetase antibody, rheumatoid factor, or anti-cyclic citrullinated peptide antibody were detected.

We initially started antibiotic therapy with ceftriaxone ( $2.0 \mathrm{~g} /$ day) because of the possibility of bacterial pleurisy as indicated by the clinical symptoms and existence of the pleural effusion. At the same time, because we suspected RF based on his radiological findings, we discontinued the aspirin he was taking to prevent a recurrence of cerebral infarction and began heparin bridging therapy before performing a biopsy on this patient to obtain a definitive diagnosis. After 1 week, the patient's condition improved slightly, his CRP level decreased to $10.15 \mathrm{mg} / \mathrm{dL}$, and antibiotic therapy was thus stopped. We then performed a CT-guided biopsy of the mass lesion surrounding the abdominal aorta (Fig. 2). The biopsy samples from the retroperitoneal mass showed lymphoplasmacytic infiltration and fibrosis, and the infiltration of IgG4+ plasma cells with a ratio of $\mathrm{IgG} 4+/ \mathrm{IgG}+$ cells $>$
50\%. Storiform fibrosis was also present (Fig. 3). Therefore, we diagnosed the patient to have IgG4-related RF. During this hospitalization for the biopsy, his initial symptoms (including fever, cough, and right-sided chest pain) improved, and his serum CRP level decreased to $2.51 \mathrm{mg} / \mathrm{dL}$ on the 14th hospital day. In addition, as there was no apparent worsening of his kidney disease, he was therefore discharged from the hospital. Immediately after discharge, he underwent an ${ }^{18} \mathrm{~F}$-fluorodeoxyglucose (FDG)-positron emission tomograph (PET)/CT scan, which showed a mild FDG uptake [standardized uptake value (SUV)max 3.5] within the retroperitoneal mass, but no other suspicious findings of IgG4-related disease. Therefore, we considered the possibility of this patient having both IgG4-related pleurisy as the cause of his pleural effusion and IgG4-related kidney disease as the cause of his CKD to be low, and thus we diagnosed RF as a single IgG4-related lesion.

At 2 months following the diagnosis of IgG4-related RF, he had no complaints, and his CRP level had decreased to within the normal range, and his IgG4 had also decreased to $41 \mathrm{mg} / \mathrm{dL}$. Moreover, radiological findings showed an improvement of the retroperitoneal lesions (Fig. 1D-F). He recently underwent a follow-up outpatient examination that showed no relapse of RF for 10 months.

\section{Discussion}

$\mathrm{RF}$ is a rare syndrome hallmarked by fibrosclerotic tissue in the retroperitoneum, often leading to encasement of the ureters (5). RF is generally divided into two types: idiopathic RF, for which no clear cause is known, and secon- 

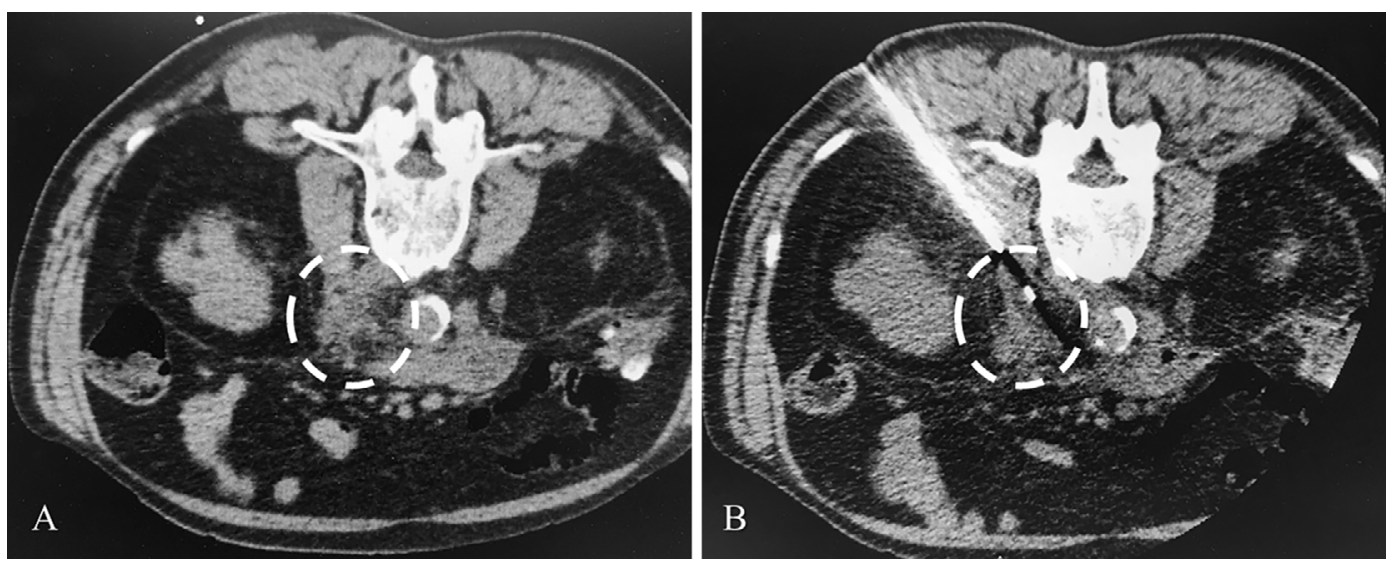

Figure 2. Computed tomography (CT)-guided percutaneous needle biopsy for muddiness of the retroperitoneal mass lesion (dotted circle). (A) Marking of the biopsy entry site on the CT image in the prone position. (B) CT guided percutaneous fine needle aspiration biopsies using 18G cutting needles.
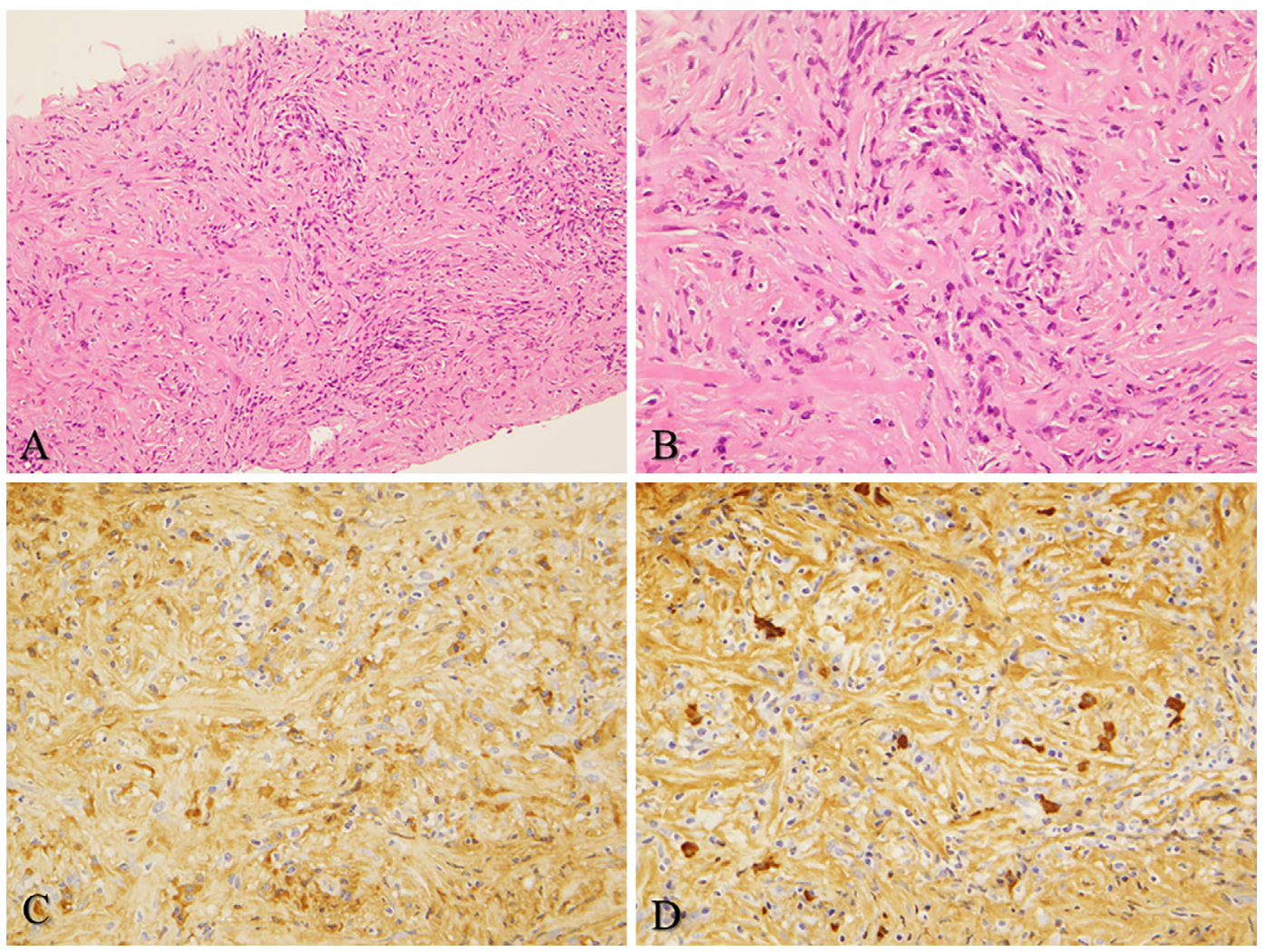

Figure 3. Histopathological findings of the retroperitoneal mass lesion. Lymphoplasmacytic infiltration and fibrosis were seen (A) [Hematoxylin and Eosin $(\mathrm{H} \& \mathrm{E})$ staining, $\times 200]$. Storiform fibrosis was also present $(\mathrm{B})(\mathrm{H} \& \mathrm{E}$ staining, $\times 400)$. IgG immunohistochemical staining revealed that most of the infiltrating plasma cells were positive $(\times 400)$. IgG4 immunohistochemical staining revealed that more than $50 \%$ of the IgG-positive plasma cells were positive for IgG4 $(\times 400)$.

dary RF, which occurs secondary to such causes as drug therapy, malignant tumors, and chronic infections (e.g., tuberculosis, histoplasmosis, actinomycosis) $(1,5)$. Idiopathic RF often shows manifestations of an autoimmune disease and it has been found to occasionally occur as an extrapancreatic lesion of autoimmune pancreatitis. More recently, some forms of idiopathic RF are considered to be a part of the spectrum of IgG4-related disease (5-9), which has newly been recognized to be a fibro-inflammatory condition characterized by several features: a tendency to form tumefactive lesions at multiple sites, a characteristic histopathological appearance, and often, but not always, an elevated serum IgG4 concentration (10). Umehara et al. reported the comprehensive diagnostic criteria for IgG4-related disease as fol- 
lows: 1. clinical examination showing characteristic diffuse/ localized swelling or masses in single or multiple organs; 2. An elevated serum IgG4 concentration (>135 mg/dL); and 3 . A histopathological examination showing marked lymphocyte and plasmacytic infiltration and fibrosis, and the infiltration of $\mathrm{IgG} 4+$ plasma cells of $>40 \%$ and $>10$ IgG4+ plasma cells/HPF (11). Based on these criteria, we confirmed the definitive diagnosis of IgG4-related disease in our patient. Koo et al. reported that among patients with $\mathrm{IgG} 4$ related RF, all had dense lymphoplasmacytic infiltrations, $88.9 \%$ of them showed storiform fibrosis, and $44.4 \%$ had obliterative phlebitis (6). At the time our patient received antibiotic therapy for acute infectious pleurisy, it was possible that this therapy had an effect on the etiology of RF. However, our patient was diagnosed as having idiopathic IgG4related RF that showed the typical pathological features. There were no other findings of secondary RF due to drug therapy, malignant tumors, or chronic infections.

The clinical course of our patient is considered to have important clinical implications: IgG4-related RF can spontaneously remit without any treatment. In fact, unexpectedly, a spontaneous remission of his clinical symptoms and radiological abnormalities associated with IgG4-related RF occurred during hospitalization. To the best of our knowledge, there is only one other reported case of a spontaneous remission of IgG4-related RF. Miura and Miyachi reported a patient who showed a spontaneous remission of IgG4related RF, although it recurred 5 years after remission (12). However, they diagnosed the patient to have IgG4-related RF because the serum IgG4 levels strongly correlated with the disease behavior of RF; however, this case was not biopsy proven. Therefore, the present report describes what we believe to be the first case of a spontaneous regression of biopsy proven IgG4-related RF.

The precise mechanism of the spontaneous remission in our patient is unclear. In IgG4-related disease, serum autoantibodies including anti-lactoferrin, rheumatoid factor, allergic disorder, and elevated levels of serum IgE are often detected, and therefore the pathogenesis of IgG4-related disease is generally assumed from autoimmune and allergic responses $(13,14)$. In terms of autoimmune response, although no specific antigen targets for the IgG4 antibodies have been identified in patients with IgG4-related disease, IgG4 antibodies can activate both complements via the alternate pathway and leukocytes and can induce leukocytedependent tissue damage (15). With regard to allergic response, there is a known association between the preferential Th2-type response and an increase in the expression of the Th2 cytokines IL-4, IL-5, IL-10, and IL-13. Activated regulatory $\mathrm{T}$ cells secrete transforming growth factor- $\beta$, which is responsible for the fibrosis seen in IgG4-related disease (16). We surmise that one of the reasons for this is that all types of cytokines might be regulated for an immune and allergic self-limiting response. In fact, some cases of a spontaneous regression of IgG4-related pulmonary and hepatic inflammatory pseudotumor have been reported similar to our case $(17,18)$.

In clinical practice, the first goal of treatment in RF is the relief of ureteral obstruction to prevent any significant impairment in the function of one or both kidneys. Recently, a conservative approach followed by the administration of medical therapy (e.g., steroids) has become popular (19), and therefore, the long-term disease behavior of untreated IgG4-related RF remains unknown. However, our patient spontaneously showed complete remission during the clinical course. Importantly, IgG4-related RF rapidly responds to steroid treatment $(3,8)$. Taking the long-term adverse effects of steroid therapy together with the rapid response to steroid treatment, careful observation to determine whether or not the renal function is worsening in preparation for initiating steroid treatment may be one important treatment strategy in asymptomatic IgG4-related RF patients without IgG4-related kidney disease. However, because the natural history may be unclear, care should be taken to check for any frequent relapse of $\operatorname{IgG} 4$-related disease over the long term $(12,20)$.

In conclusion, we herein described the first known case of a spontaneous remission of biopsy proven IgG4-related RF. Because RF is essentially a rare disease, and the concept of IgG4-related disease is unfamiliar to many clinicians, few studies have been published on IgG4-related RF. Although the pathogenesis and long-term disease behavior of IgG4related RF remains unknown, it will be important to continue to accumulate data and evaluate many cases of IgG4related RF and identify the optimal therapeutic strategies.

The authors state that they have no Conflict of Interest (COI).

\section{References}

1. Vaglio A, Palmisano A, Corradi D, Salvarani C, Buzio C. Retroperitoneal fibrosis: evolving concepts. Rheum Dis Clin North Am 33: 803-817, 2007

2. Vaglio A, Salvarani C, Buzio C. Retroperitoneal fibrosis. Lancet 367: 241-251, 2006.

3. Fujimori N, Ito T, Igarashi H, et al. Retroperitoneal fibrosis associated with immunoglobulin G4-related disease. World J Gastroenterol 19: 35-41, 2013.

4. Vaglio A, Palmisano A, Alberici F, et al. Prednisone versus tamoxifen in patients with idiopathic retroperitoneal fibrosis: an open-label randomised controlled trial. Lancet 378: 338-346, 2011.

5. Pipitone N, Vaglio A, Salvarani C. Retroperitoneal fibrosis. Best Pract Res Clin Rheumatol 26: 439-448, 2012.

6. Koo BS, Koh YW, Hong S, et al. Clinicopathologic characteristics of IgG4-related retroperitoneal fibrosis among patients initially diagnosed as having idiopathic retroperitoneal fibrosis. Mod Rheumatol 25: 194-198, 2015.

7. Corradi D, Maestri R, Palmisano A, et al. Idiopathic retroperitoneal fibrosis: clinicopathologic features and differential diagnosis. Kidney Int 72: 742-753, 2007.

8. Zen Y, Onodera M, Inoue D, et al. Retroperitoneal fibrosis: a clinicopathologic study with respect to immunoglobulin G4. Am J Surg Pathol 33: 1833-1839, 2009.

9. Mahajan VS, Mattoo H, Deshpande V, Pillai SS, Stone JH. IgG4related disease. Annu Rev Pathol 9: 315-347, 2014.

10. Deshpande V, Zen Y, Chan JK, et al. Consensus statement on the pathology of IgG4-related disease. Mod Pathol 25: 1181-1192, 
2012.

11. Umehara H, Okazaki K, Masaki $Y$, et al. Comprehensive diagnostic criteria for IgG4-related disease (IgG4-RD), 2011. Mod Rheumatol 22: 21-30, 2012.

12. Miura H, Miyachi Y. IgG4-related retroperitoneal fibrosis and sclerosing cholangitis independent of autoimmune pancreatitis. A recurrent case after a 5-year history of spontaneous remission. JOP 10: 432-437, 2009.

13. Okazaki K, Uchida K, Ohana M, et al. Autoimmune-related pancreatitis is associated with autoantibodies and a Th1/Th2-type cellular immune response. Gastroenterology 118: 573-581, 2000.

14. Masaki Y, Dong L, Kurose N, et al. Proposal for a new clinical entity, IgG4-positive multiorgan lymphoproliferative syndrome: analysis of 64 cases of IgG4-related disorders. Ann Rheum Dis 68: 1310-1315, 2009.

15. Fernandez-Flores A. The role of IgG4 in cutaneous pathology. Rom J Morphol Embryol 53: 221-231, 2012.

16. Raj R. IgG4-related lung disease. Am J Respir Crit Care Med 188: 527-529, 2013
17. Sakashita K, Takamori M, Murata K, Wada A, Fujita A, Enatsu K. A case of IgG4-positive inflammatory pseudotumor which rapidly resolved. Nihon Kokyuki Gakkai Zasshi (Ann Jpn Respir Soc) 49: 172-177, 2011.

18. Yamaguchi J, Sakamoto Y, Sano T, Shimada K, Kosuge T. Spontaneous regression of inflammatory pseudotumor of the liver: report of three cases. Surg Today 37: 525-529, 2007.

19. van Bommel EF, Siemes C, Hak LE, van der Veer SJ, Hendriksz TR. Long-term renal and patient outcome in idiopathic retroperitoneal fibrosis treated with prednisone. Am J Kidney Dis 49: 615625, 2007.

20. Cheuk W, Chan JK. IgG4-related sclerosing disease: a critical appraisal of an evolving clinicopathologic entity. Adv Anat Pathol 17: $303-332,2010$

The Internal Medicine is an Open Access article distributed under the Creative Commons Attribution-NonCommercial-NoDerivatives 4.0 International License. To view the details of this license, please visit (https://creativecommons.org/licenses/ by-nc-nd/4.0/).

(C) 2017 The Japanese Society of Internal Medicine http://www.naika.or.jp/imonline/index.html 